Der Weg zum Nationalen Allokationsplan in Deutschland

\section{Ein fauler Kompromiss}

\section{Die erst im Herbst 2003 in Kraft getretene EU-Emissionshandelsrichtlinie setzt den EU-Mitgliedstaaten sehr enge Fristen zur nationalen Umsetzung, da der europaweite Handel mit Emissionsrechten für Kohlendioxid bereits zum ersten Januar 2005 beginnen soll. Zentrales Element zur Umsetzung der Richtlinie ist der Nationale Allokationsplan, in dem jeder EU-Mitgliedstaat für jede Handels- periode die Kohlendioxid-Obergrenzen für die am Emissionshandel beteiligten Anlagen, die Zuteilung der Emissionsrechte, aber auch Politiken und Maß- nahmen für die nicht am Emissionshandel beteiligten Sektoren darzulegen hat.}

$\mathrm{N}$ Von Mario Meinecke achdem diverse Verhandlungsrunden bis Ende Januar 2004 zu keiner Konkretisierung der Ausgestaltung des deutschen Nationalen Allokationsplans (NAP) geführt hatten, legte das Bundesumweltministerium (BMU) am 29. Januar einen NAP-Entwurf vor, der - so Bundesumweltminister Trittin - der Wirtschaft beziehungsweise den am Emissionshandel beteiligten Sektoren bis 2012 nicht mehr Kohlendioxid-Reduktion abverlangt, als sie bereits in Selbstverpflichtungen zugesagt haben. Grundlage für den in den folgenden zwei Monaten heftig umstrittenen NAP-Entwurf war die Selbstverpflichtung zur Kraft-Wärme-Kopplung (KWK), die am 19. Dezember 2003 in Kraft getreten ist. Darin sichern die beteiligten Industrieverbände im Vergleich zu 1998 eine absolute Verringerung ihrer Kohlendioxid-Emissionen bis 2005 um 20 Millionen Tonnen und bis 2010 um 45 Millionen Tonnen zu, davon 10 Millionen Tonnen in ,anderen Sektoren“.

Der NAP-Entwurf des BMU nahm die noch nicht einmal zwei Monate alte KWK-Vereinbarung beim Wort und setzte für die Sektoren Industrie und Energiewirtschaft für die erste Handelsperiode von Kohlendioxid-Zertifikaten (2005 bis 2007) ein jährliches Kohlendioxid-Emissionsbudget von 488 Millionen Tonnen und für die zweite Handelsperiode (2008 bis 2012) von 480 Millionen Tonnen Kohlendioxid fest. Da in diesen beiden Sektoren 1998508 Millionen Tonnen Kohlendioxid emittiert worden sind, hätte dies für den Zeitraum 2005 bis 2007 eine Reduktion um 20 Millionen und für den Zeitraum 2008 bis 2012 um 28 Millionen Tonnen bedeutet.

\section{Reduktionsverpflichtungen}

Die gesamten Kohlendioxid-Emissionen in Deutschland betrugen im Durchschnitt der Jahre 2000 bis 2002, die als Grundlage für den NAP dienen, 863 Millionen Tonnen. Zur Erreichung des Kioto-Ziels von 846 Millionen Tonnen Kohlendioxid 2008 beziehungsweise 2012 wäre also 17 Millionen Tonnen Kohlendioxid zu erbringen. Somit hätten die Sektoren Industrie und Energiewirtschaft nach dem BMU-Entwurf des NAP die noch ausstehende Reduktionsverpflichtung nahezu allein zu erbringen gehabt, während den Sektoren Handel/Kleingewerbe/Dienstleistungen (minus drei Millionen Tonnen 2008/2012 im Vergleich zu 2000/2002), Verkehr (plus sechs Millionen Tonnen) und private Haushalte (plus fünf Millionen Tonnen) bis 2012 kaum KohlendioxidReduktionsverpflichtungen beziehungsweise sogar Steigerungen ,abverlangt“ worden wären. Das mutet zunächst wenig ambitioniert an, ist jedoch vor dem Hintergrund einer Referenzentwicklung ohne weitere umweltpolitische Maßnahmen zu sehen. Den Kohlendioxid-Reduktionseffekt der von der Bundesregierung seit 1998 bereits ergriffenen Maßnahmen in den Sektoren Verkehr und private Haushalte wie etwa der ökologischen Steuerreform, der Förderung von Biokraftstoffen, der Lkw-Maut, KfW-Programme zur Gebäudemodernisierung und zur Wärmedämmung gibt das BMU mit insgesamt rund 25 Millionen Tonnen Kohlendioxid an. Mit weiteren, noch zu ergreifenden Maßnahmen wären im Vergleich zur Referenzentwicklung bis 2012 weitere rund 13 Millionen Tonnen Kohlendioxid einzusparen. Dennoch wären laut NAP-Entwurf des BMU die gesamtgesellschaftlich noch eine Reduktion von oben genannten Steigerungen in den Emissionsbudgets für die Sektoren Haushalte und Verkehr bis 2012 unumgänglich. Da liegt es nahe, die KWK-Selbstverpflichtungserklärung heranzuziehen und die zur Erfüllung des Kioto-Protokolls notwendigen Kohlendioxid-Reduktionen durch die Sektoren Industrie und Energieerzeugung erbringen zu lassen, aus denen der überwiegende Anteil der rund 2.400 deutschen Anlagen stammt, die am Emissionshandel teilnehmen.

\section{Wirtschaft blockiert}

Es zeigte sich allerdings sehr schnell, dass weder Bundeswirtschaftsminister Clement noch die am Emissionshandel beteiligten Industriesektoren mit dem NAP-Entwurf des BMU einverstanden waren. Beteuerungen des BMU, der NAP-Entwurf enthalte keine Verpflichtungen für Industrie und Energiewirtschaft, die über ihre eigenen Kohlendioxid -Reduktionszusagen hinausgingen, blieben ungehört; an die KWK-Selbstverpflichtungsvereinbarung schien sich plötzlich niemand mehr erinnern zu können. Die Debatte wurde sehr scharf geführt und war außerordentlich vielstimmig, weil jedes Unternehmen beziehungsweise jeder Anlagenbetreiber ein eigenes Interesse im komplizierten Geflecht des Emissionshandels hatte. Vielleicht gerade wegen dieser vielschichtigen Interessenlage waren weder das Bundesministerium für Wirtschaft (BMWA) noch die Industrieverbände Willens oder in der Lage, sich mit dem vorliegenden NAP-Entwurf auseinanderzusetzen. Auf schriftliche Stellungnahmen aus dem BMWA wartete man noch vier Wochen nach Veröffentlichung des BMU-Entwurfs vergeblich, während der Bundesverband der Deutschen Industrie (BDI) Vorschläge präsentierte, die eine Ausweitung der Kohlendioxid-Emissionen statt deren Reduktion bedeutet hätten. Der BDI empfahl seinen Mitgliedsunternehmen sogar, die Debatte ,holzschnittartig" zu führen und sich keinesfalls auf eine inhaltliche Debatte einzulassen.

Das Verhältnis zwischen den beteiligten Ministerien war derart zerrüttet, dass die Minister Clement und Trittin nur noch schriftlich mit einander korrespondierten. Mitte März ließ Clement sogar einen von Kanzleramtsminister Steinmeier mit den Staatssekretären beider Ministerien in nächtlicher Sitzung ausgehandelten Kompromiss am nächsten Morgen mit seinem Veto scheitern. In der Nacht vom 29. auf den 30. März 2004 eineinhalb Tage vor Abgabefrist des NAP an die EU-Kommission - kam es dann bei einem Spitzengespräch, an dem auch Bundeskanzler Schrö- 
der und Außenminister Fischer teilnahmen, doch noch zu einem Kompromiss zwischen den Ministern Clement und Trittin. Auf der Grundlage dieser Einigung, die Umweltminister Trittin nur Zähne knirschend akzeptierte, verabschiedete das Bundeskabinett am 31. März einen Nationalen Allokationsplan für die erste Handelsperiode von 2005 bis 2007, der doch noch rechtzeitig der EUKommission übermittelt werden konnte.

Da die engen Zeitvorgaben der EU-Richtlinie bis zur Übermittlung des NAP an die EU-Kommission keine parlamentarische Beteiligung zugelassen haben, hat die Bundesregierung den NAP mit einem Parlamentsvorbehalt versehen. Die endgültige Entscheidung über die Emissionsobergrenzen der einzelnen Sektoren und über die Grundzüge der Zuteilung der Kohlendioxid-Emissionshandelszertifikate bleibt also dem Deutschen Bundestag vorbehalten. Noch im April wurde der Entwurf eines NAP-Gesetzes in den Bundestag eingebracht, der schon Ende Mai mit zahlreichen Detailänderungen in zweiter und dritter Lesung verabschiedet wurde.

Der von der Bundesregierung verabschiedete NAP, weicht vom ursprünglichen Entwurf des BMU erheblich ab (1). Den Sektoren Industrie und Energiewirtschaft wird für 2005 bis 2007 zusammen ein jährliches Emissionsbudget von 503 Millionen Tonnen Kohlendioxid und für 2008 bis 2012 von 495 Millionen Tonnen Kohlendioxid zugebilligt. Dies bedeutet im Vergleich zum Durchschnitt der Jahre 2000 bis 2002 (505 Millionen Tonnen Kohlendioxid) lediglich eine Reduktion von zwei Millionen für 2005 bis 2007 und 10 Millionen Tonnen für 2008 bis 2012 .

Da das nationale Kohlendioxid-Emissionsbudget bis 2012 durch das Kioto-Protokoll und den verbindlich zugesagten Anteil Deutschland am EUburden sharing vorgegeben ist, muss die Differenz zum BMU-Entwurf des NAP von 15 Millionen Tonnen Kohlendioxid, die laut Kabinettsbeschluss nun nicht mehr von den Sektoren Industrie und Energiewirtschaft erbracht werden soll, von den übrigen Sektoren (Haushalte, Verkehr und Handel/Kleingewerbe/Dienstleistungen) erbracht werden.

\section{- Zukunft ungeklärt}

Welche zusätzlichen Maßnahmen die Bundesregierung ergreifen will, um in diesen Sektoren im Vergleich zum NAP-Entwurf des BMU, der bereits zahlreiche Maßnahmen enthielt, bis 2012 weitere 15 Millionen Tonnen Kohlendioxid einzusparen, bleibt offen. Wenn die Ökologische Steuerreform bisher etwa zu einer Einsparung von fünf Millionen Tonnen Kohlendioxid geführt hat und für eine funktionierende Lkw-Maut etwa ein Einsparpotenzial von zwei Millionen Tonnen Kohlendioxid veranschlagt wird, dann entspräche die Reduktion von 15 Millionen Tonnen Kohlendioxid in den Sektoren Haushalte, Kleingewerbe und Verkehr etwa einer Vervierfachung der Ökologischen Steuerreform bis 2012 oder einer Verachtfachung der Lkw-Maut - wenn sie denn einmal eingeführt ist. Alternativ müsste die Regierung ein mehrere Milliarden Euro schweres Programm zur Kohlendioxid-Gebäudesanierung auflegen, um einen derartigen KohlendioxidEinspareffekt zu erzielen. Wie wahrscheinlich das Ergreifen einer dieser Maßnahmen oder eine entsprechende Kombination ist, mag sich jede/r selbst ausmalen.

Vor dem Hintergrund, dass Deutschland mit dem Kioto-Protokoll und dem EU-burden sharing international verbindliche Verpflichtungen zur Kohlendioxid-Reduktion eingegangen ist, wird sich allerdings kein Politiker vorwerfen lassen wollen, er hätte das Verfehlen der Kioto-Zielvereinbarung sehenden Auges wirtschaftlichen oder kurzfristigen politischen Interessen geopfert. Ohne den politischen Mut zu unpopulären und kostspieligen umweltpolitischen Maßnahmen ist es allerdings sehr unwahrscheinlich, dass die durch den NAP vorgegebenen KohlendioxidEinsparziele in den Sektoren private Haushalte und/oder Verkehr bis 2012 erreicht werden. Der Kabinettsbeschluss zum NAP macht damit das Erreichen des Kioto-Ziels eher schwieriger als einfacher. Wenn man am Kioto-Ziel festhält, könnte das schließlich bedeuten, dass den Sektoren Industrie und Energiewirtschaft - und damit den am Emissionshandel beteiligten Anlagen - bis 2012 doch noch eine höhere Kohlendioxid-Reduktionsverpflichtung abverlangt werden muss, als im derzeitigen NAP vorgesehen. Wie wenig ambitioniert das im NAP verankerte Kohlendioxid-Reduktionsziel für die Sektoren Industrie und Energiewirtschaft ist, zeigt das folgende Beispiel:

In den Kohlendioxid-Emissionen der Jahre 20002002, die als Basis für die Verteilung der Emissionszertifikate dienen, ist die Inbetriebnahme des modernsten und effizientesten Braunkohlekraftwerks BoA (Braunkohlekraftwerk mit optimierter Anlagentechnik) in Niederaußem noch nicht enthalten, das zwar 2002 fertig gestellt aber erst später hochgefahren wurde - als Ersatz für sechs kleinere Braunkohle-Kraftwerksblöcke aus den 1960er Jahren.

\section{Ausblick}

Alleine durch das Hochfahren dieses Kraftwerks und das parallele Abschalten der alten, ineffizienten Blöcke werden mindestens 2,5 Millionen Tonnen Kohlendioxid pro Jahr eingespart 500.000 Tonnen mehr als die Sektoren Industrie und Energieversorgung laut NAP bis 2007 einsparen müssen. Das Ziel ist - gleich bleibende Produktion vorausgesetzt - also schon jetzt übererfüllt. Es drängt sich förmlich der Verdacht auf, dass das langfristig angelegte klimapolitische Ziel kurzfristigen politischen Interessen geopfert wurde. Hierfür gibt es zwei Hauptgründe.

Ohne dass es je öffentlich ausgesprochen wurde, galt es als undenkbar, dass mit EEG-Novelle und Emissionshandel, die beide zeitgleich im Frühjahr 2004 auf der politischen Agenda standen, zwei Themen als umweltpolitischer Erfolg gegen den Widerstand von Minister Clement verabschiedet werden können. Da starke Beschneidungen am umwelt- und wirtschaftspolitisch erfolgreichen EEG vor der internationalen Konferenz renewables 2004 Anfang Juni in Bonn aber als Tabu galten, blieb der NAP als Faustpfand des Wirtschaftsministers. Ein zweiter Grund für einen industrie- und kohlefreundlichen Nationalen Allokationsplan zumindest für die erste Handelsperiode von Emissionszertifikaten ist die Landtagswahl im Frühjahr 2005 in Nordrhein-Westfalen - für die SPD der entscheidende Stimmungstest vor der Bundestagswahl 2006. Zufällig war Clement dort 1998 bis 2002 Ministerpräsident.

\section{Anmerkung}

(1) Für nähere Informationen siehe: www.bmu.de/de/ 1024/is/sachthemen/emissionshandel/nap/ sowie die Bundestags-Drucksachen-Nummern 15/2966 (Entwurf) und 15/3224 (Gesetz). 
(c) 20I0 Authors; licensee IÖW and oekom verlag. This is an article distributed under the terms of the Creative Commons Attribution Non-Commercial No Derivates License (http://creativecommons.org/licenses/by-nc-nd/3.o/), which permits unrestricted use, distribution, and reproduction in any medium, provided the original work is properly cited. 